\title{
LINEAR MAPS PRESERVING THE HIGHER NUMERICAL RANGES OF TENSOR PRODUCTS OF MATRICES
}

\author{
AJDA FOŠNER, ZEJUN HUANG, CHI-KWONG LI, YIU-TUNG POON, AND NUNG-SING SZE
}

\section{Dedicated to Professor Natalia Bebiano on the occasion of her birthday.}

\author{
Abstract. For a positive integer $n$, let $M_{n}$ be the set of $n \times n$ complex matrices. Suppose $m, n \geq 2$ \\ are positive integers and $k \in\{1, \ldots, m n-1\}$. Denote by $W_{k}(X)$ the $k$-numerical range of a matrix \\ $X \in M_{m n}$. It is shown that a linear map $\phi: M_{m n} \rightarrow M_{m n}$ satisfies

$$
W_{k}(\phi(A \otimes B))=W_{k}(A \otimes B)
$$ \\ for all $A \in M_{m}$ and $B \in M_{n}$ if and only if there is a unitary $U \in M_{m n}$ such that one of the following \\ holds. \\ (i) For all $A \in M_{m}, B \in M_{n}$,

$$
\phi(A \otimes B)=U(\varphi(A \otimes B)) U^{*} .
$$ \\ (ii) $m n=2 k$ and for all $A \in M_{m}, B \in M_{n}$,

$$
\phi(A \otimes B)=(\operatorname{tr}(A \otimes B) / k) I_{m n}-U(\varphi(A \otimes B)) U^{*},
$$ \\ where (1) $\varphi$ is the identity map $A \otimes B \mapsto A \otimes B$ or the transposition map $A \otimes B \mapsto(A \otimes B)^{t}$, or \\ (2) $\min \{m, n\} \leq 2$ and $\varphi$ has the form $A \otimes B \mapsto A \otimes B^{t}$ or $A \otimes B \mapsto A^{t} \otimes B$.
}

2010 Math. Subj. Class.: 15A69, 15A86, 15A60, 47A12.

Key words: Hermitian matrix, linear preserver, $k$-numerical range, tensor product of matrices.

\section{INTRODUCTION AND THE MAIN THEOREM}

Let $M_{n}\left(H_{n}\right)$ be the set of $n \times n$ complex (Hermitian) matrices. For $k \in\{1, \ldots, n\}$, the $k$ numerical range of $A \in M_{n}$ is defined as

$$
W_{k}(A)=\left\{\operatorname{tr}\left(X^{*} A X\right) / k: X \text { is } n \times k, X^{*} X=I_{k}\right\} .
$$

Since $W_{n}(A)=\{\operatorname{tr}(A) / n\}$, we always assume that $k<n$ to avoid trivial consideration. When $k=1$, we have the classical numerical range $W_{1}(A)=W(A)$, which has been studied extensively; see [9, 10, 11]. Denote by $A \otimes B$ the tensor (Kronecker) product of matrices $A \in M_{m}$ and $B \in M_{n}$. The purpose of this paper is to characterize linear maps on $M_{m n}$ satisfying

$$
W_{k}(A \otimes B)=W_{k}(\phi(A \otimes B)) \text { for all } A \in M_{m}, B \in M_{n} .
$$

The study is motivated by two areas of research.

First, in the last few decades there has been considerable interest in studying linear preservers on matrix algebras as well as on more general rings and operator algebras. For example, Frobenius [7] showed that a linear operator $\phi: M_{n} \rightarrow M_{n}$ satisfies

$$
\operatorname{det}(\phi(A))=\operatorname{det}(A)
$$

for all $A \in M_{n}$ if and only if there are $U, V \in M_{n}$ with $\operatorname{det}(U V)=1$ such that $\phi$ has the form

$$
A \mapsto U A V \quad \text { or } \quad A \mapsto U A^{t} V,
$$


where $A^{t}$ denotes the transpose of $A$. Clearly, a map of the above form is linear and leaves the determinant function invariant. But it is interesting that a linear map preserving the determinant function must be of this form. Furthermore, in 3 Dieudonné showed that a bijective linear operator $\phi: M_{n} \rightarrow M_{n}$ maps the set of singular matrices into itself if and only if there are invertible $U, V \in M_{n}$ such that $\phi$ has the standard form (2). One may see [15] and references therein for results on linear preserver problems. For more new directions and active research on preserver problems motivated by theory and applications, one may see, for example, [1, 19, 24].

In connection to the linear preservers of the $k$-numerical range, Pellegrini 21] proved that every linear map $\phi: M_{n} \rightarrow M_{n}$ preserving the numerical range is of the form

$$
A \mapsto U A U \quad \text { or } \quad A \mapsto U A^{t} U^{*}
$$

for some unitary $U \in M_{n}$. Three years later, Pierce and Watkins [22] extended this result to the $k$-numerical ranges as long as $n \neq 2 k$. In [13] (see also [20]) it was shown that for $n=2 k$, a linear map $\phi: M_{n} \rightarrow M_{n}$ preserves the $k$-numerical range if and only if there exists a unitary $U \in M_{n}$ such that $\phi$ has the form (3), or

$$
A \mapsto(\operatorname{tr}(A) / k) I_{n}-U A U^{*} \quad \text { or } \quad A \mapsto(\operatorname{tr}(A) / k) I_{n}-U A^{t} U^{*} .
$$

One may see [2, 16] for more information about the results on linear maps on $M_{n}$ which preserve the $k$-numerical range.

Another motivation of our study comes from quantum information science. In quantum physics (e.g., see [8]), quantum states are represented by density matrices $D$ in $H_{n}$, i.e., positive semidefinite matrices with trace 1 . If $D$ has rank one, i.e., $D=x x^{*}$ for some $x \in \mathbb{C}^{n}$ with $x^{*} x=1$, then $D$ is a pure state. Otherwise, $D$ is a mixed state, which can be written as a convex combination of pure states. In a quantum system, every observable corresponds to a Hermitian matrix $A$. Then

$$
W(A)=\left\{\operatorname{tr}\left(A x x^{*}\right): x \in \mathbb{C}^{n}, x^{*} x=1\right\}
$$

can be viewed as the set of all possible mean measurements of quantum states. If $A=A_{1}+i A_{2} \in$ $M_{n}$, where $A_{1}, A_{2} \in H_{n}$, then every point in $W(A)$ and be viewed as the set of all joint measurements $x^{*} A x=x^{*} A_{1} x+i x^{*} A_{2} x$ of the quantum state $x$ with respect to the two observables associated with $A_{1}$ and $A_{2}$, and thus $W(A)$ is the set of all possible joint measurements. By the convexity of $W(A)$ (e.g., see [9, 10, 11]),

$$
W(A)=\left\{\operatorname{tr}(A D): D \in H_{n} \text { is a density matrix }\right\} .
$$

Thus, $W(A)$ can also be viewed as the set of all possible joint measurements of two observables on mixed states. Now, by the convexity of $W_{k}(A)$, and the fact that the convex hull of the set $\left\{P / k: P^{2}=P=P^{*}, \operatorname{tr} P=k\right\}$ equals the set $\mathcal{S}_{k}$ of density matrices $D$ satisfying $I_{n} / k-D$ is positive semidefinite, we have

$$
W_{k}(A)=\left\{\operatorname{tr}(A P) / k: P \in H_{n}, P^{2}=P=P^{*}, \operatorname{tr} P=k\right\}=\left\{\operatorname{tr}(A D): D \in \mathcal{S}_{k}\right\} .
$$

So, $W_{k}(A)$ can be viewed as the set of joint measurements of the states in $\mathcal{S}_{k}$ with respect to the observables associated with $A$. Suppose $A \in H_{m}$ and $B \in H_{n}$ correspond to observables of two quantum systems with quantum states $D_{1} \in M_{m}$ and $D_{2} \in M_{n}$. Then the tensor (Kronecker) 
product $A \otimes B$ correspond to an observable on the joint (bipartite) system with quantum states $D \in M_{m n}$ expressed as the convex combination of uncorrelated quantum states $D_{1} \otimes D_{2}$, where $D_{1} \in M_{m}$ and $D_{2} \in M_{n}$ are quantum states (density matrices). In this connection, we are interested in studying linear maps $\phi$ on $M_{m n}$ satisfying (1).

In fact, this line of study has been carried out in [4, 5, 6, 12, 23. Suppose $f(X)$ is a linear function on the matrix $X \in M_{m n}$. It is shown in [4] that the linear maps $\phi$ on $H_{m n}$ satisfying

$$
f(\phi(A \otimes B))=f(A \otimes B)
$$

for all $A \in H_{m}$ and $B \in H_{n}$ when $f(X)$ is the spectrum or the spectral radius of $X$. In [5, 6], the authors characterized the linear maps $\phi$ on $M_{m n}$ satisfying (5) for all $A \in M_{m}$ and $B \in M_{n}$ when $f(X)$ is a Ky Fan norm, Schatten norm or the numerical radius of $X$.

The following is our main result.

Theorem 1.1. Let $k \in\{1, \ldots, m n-1\}$. A linear map $\phi: M_{m n} \rightarrow M_{m n}$ satisfies

$$
W_{k}(\phi(A \otimes B))=W_{k}(A \otimes B)
$$

for all $A \in M_{m}$ and $B \in M_{n}$ if and only if there is a unitary $U \in M_{m n}$ such that one of the following holds.

(i) For all $A \in M_{m}, B \in M_{n}$,

$$
\phi(A \otimes B)=U(\varphi(A \otimes B)) U^{*} .
$$

(ii) $m n=2 k$ and for all $A \in M_{m}, B \in M_{n}$,

$$
\phi(A \otimes B)=(\operatorname{tr}(A \otimes B) / k) I_{m n}-U(\varphi(A \otimes B)) U^{*},
$$

where (1) $\varphi$ is the identity map $A \otimes B \mapsto A \otimes B$ or the transposition map $A \otimes B \mapsto(A \otimes B)^{t}$, or (2) $\min \{m, n\} \leq 2$ and $\varphi$ has the form $A \otimes B \mapsto A \otimes B^{t}$ or $A \otimes B \mapsto A^{t} \otimes B$.

The proof of the theorem will be given in the next section. We will use the following properties of the $k$-numerical range; for example, see [10, 13, 18, 22.

Proposition 1.2. Let $A \in M_{n}$ and $k \in\{1, \ldots, n-1\}$.

- For any $\alpha, \beta \in \mathbb{C}, W_{k}(A)=\{\alpha\}$ if and only if $A=\alpha I_{n}$, and $W_{k}\left(\alpha I_{n}+\beta A\right)=\alpha+\beta W_{k}(A)$.

- For any unitary $U \in M_{n}, W_{k}\left(U A U^{*}\right)=W_{k}(A)$.

- For any $s \times n$ matrix $V$ with $s \geq k$ and $V V^{*}=I_{s}$, we have $W_{k}\left(V A V^{*}\right) \subseteq W_{k}(A)$.

- $W_{k}(A) \subseteq \mathbb{R}$ if and only if $A$ is Hermitian.

- If $A \in H_{n}$ has eigenvalues $\alpha_{1} \geq \cdots \geq \alpha_{n}$, then

$$
W_{k}(A)=\left[\left(\alpha_{n-k+1}+\cdots+\alpha_{n}\right) / k,\left(\alpha_{1}+\cdots+\alpha_{k}\right) / k\right] .
$$

- $W_{k}\left(\frac{A+A^{*}}{2}\right)=\operatorname{Re}\left(W_{k}(A)\right) \equiv\left\{\operatorname{Re}(z): z \in W_{k}(A)\right\}$.

To conclude our introduction, let us point out that we consider only the bipartite case, i.e., $M_{m} \otimes M_{n}$ with integers $m, n \geq 2$. Our proofs are rather technical and we are not able to extend 
them to the multipartite systems $M_{n_{1}} \otimes \cdots \otimes M_{n_{m}}$ with $n_{1}, \ldots, n_{m} \geq 2$ and $m>2$. Furthermore, one can define the $k$-numerical radius of a square matrix $A \in M_{n}$ by

$$
w_{k}(A)=\max \left\{|x|: x \in W_{k}(A)\right\} .
$$

It would also be interesting to characterize the linear preservers of the $k$-numerical radius on the bipartite or multipartite systems. Again, it does not seem easy to apply our proofs to solve this problem.

\section{Proof of Theorem 1.1}

In the following, denote by $E_{i j} \in M_{n}, 1 \leq i, j \leq n$, the matrix whose $(i, j)$-entry is equal to one and all the others are equal to zero. Two matrices $A, B \in M_{n}$ are called orthogonal if $A B^{*}=A^{*} B=0$. We write $A \perp B$ to indicate that $A$ and $B$ are orthogonal. It is shown in [17 that $A \perp B$ if and only if there are unitary matrices $U, V \in M_{n}$ such that $U A V=\operatorname{Diag}\left(\alpha_{1}, \ldots, \alpha_{n}\right)$ and $U B V=\operatorname{Diag}\left(\beta_{1}, \ldots, \beta_{n}\right)$ with $\alpha_{i}, \beta_{i} \geq 0$ and $\alpha_{i} \beta_{i}=0$ for $i=1, \ldots, n$. The matrices $A_{1}, \ldots, A_{s}$ are said to be pairwise orthogonal if $A_{i}^{*} A_{j}=A_{i} A_{j}^{*}=0$ for any distinct $i, j \in\{1, \ldots, s\}$. In this case, there are unitary matrices $U, V \in M_{n}$ such that $U A_{i} V=D_{i}$ for $i=1, \ldots, s$ with each $D_{i}$ being nonnegative diagonal matrix and $D_{i} D_{j}=0$ for any distinct $i, j \in\{1, \ldots, s\}$. We will need the following lemmas in the proof. The first lemma was proved in [14].

Lemma 2.1. [14, Lemma 4.1] Let $k \in\{1, \ldots, n\}$ and suppose $A \in H_{n}$ have diagonal entries $a_{1}, \ldots, a_{n}$ and eigenvalues $\lambda_{1} \geq \cdots \geq \lambda_{n}$ respectively. Then $\sum_{j=1}^{k} a_{j}=\sum_{j=1}^{k} \lambda_{j}$ if and only if $A=A_{1} \oplus A_{2}$ where $A_{1} \in H_{k}$ has eigenvalues $\lambda_{1}, \ldots, \lambda_{k}$.

Lemma 2.2. Let $k \in\{1, \ldots, n\}$ and $A, B \in H_{n}$ be positive semidefinite matrices. Suppose $\operatorname{tr}(A) / k=\max \left\{x: x \in W_{k}(A-B)\right\}$. Then $A \perp B$.

Proof. Suppose $U \in M_{n}$ is a unitary matrix such that $U(A-B) U^{*}=\operatorname{Diag}\left(\lambda_{1}, \ldots, \lambda_{n}\right)$ with $\lambda_{1} \geq \cdots \geq \lambda_{n}$. Denote the diagonal entries of $U A U^{*}$ and $U B U^{*}$ by $a_{1}, \ldots, a_{n}$ and $b_{1}, \ldots, b_{n}$, respectively. Then $a_{i}, b_{i}$ are nonnegative for $i=1, \ldots, n$, since $A \geq 0$ and $B \geq 0$. Now

$$
\sum_{i=1}^{k}\left(a_{i}-b_{i}\right)=\sum_{i=1}^{k} \lambda_{i}=\operatorname{tr}(A)
$$

leads to

$$
\sum_{i=1}^{k} a_{i}=\operatorname{tr}(A), \quad a_{k+1}=\cdots=a_{n}=0 \quad \text { and } \quad b_{1}=\cdots=b_{k}=0 .
$$

Using the fact $A \geq 0$ and $B \geq 0$ again, $U A U^{*}$ and $U B U^{*}$ must have the form

$$
U A U^{*}=A_{1} \oplus 0_{n-k}, \quad U B U^{*}=0_{k} \oplus B_{1}
$$

which means $A \perp B$.

Denote by $\lambda_{1}(X) \geq \cdots \geq \lambda_{n}(X)$ the eigenvalues of a Hermitian matrix $X \in M_{n}$.

Lemma 2.3. Let $k \in\{1, \ldots, m n-1\}$ and $\phi: M_{m n} \rightarrow M_{m n}$ be a linear map satisfying (6). The following conditions hold. 
(a) $\phi\left(H_{m n}\right) \subseteq H_{m n}$.

(b) $\phi\left(I_{m n}\right)=I_{m n}$.

(c) $\phi$ is trace-preserving. Furthermore, if $m n \neq 2 k$, then $\phi\left(E_{i i} \otimes E_{j j}\right)$ is positive semidefinite for all $1 \leq i \leq m$ and $1 \leq j \leq n$.

Proof. (a) Let $A \in H_{m}$ and $B \in H_{n}$. Then $W_{k}(\phi(A \otimes B))=W_{k}(A \otimes B) \subseteq \mathbb{R}$. By Proposition 1.2. $\phi(A \otimes B) \in H_{m n}$. Since every $C \in H_{m n}$ is a linear combination of matrices of the form $A \otimes B$ with $A \in H_{m}$ and $B \in H_{n}$, we see that $\phi$ maps $H_{m n}$ to $H_{m n}$.

(b) $W_{k}\left(\phi\left(I_{m n}\right)\right)=W_{k}\left(I_{m n}\right)=\{1\}$. Thus, $\phi\left(I_{m n}\right)=I_{m n}$.

(c) Let $\alpha_{1} \geq \cdots \geq \alpha_{m n}$ be eigenvalues of $\phi\left(E_{i i} \otimes E_{j j}\right)=A_{i j}$. Since $W_{k}\left(A_{i j}\right)=W_{k}\left(E_{i i} \otimes E_{j j}\right)=$ $[0,1 / k]$, we have $\alpha_{1}+\cdots+\alpha_{k}=1$ and $\alpha_{m n-k+1}+\cdots+\alpha_{m n}=0$. If $m n=2 k$, then $\operatorname{tr} A_{i j}=1+0=1$.

If $m n>2 k$, then $\alpha_{k+1}, \ldots, \alpha_{m n-k} \geq \alpha_{m n-k+1} \geq 0$ and, thus, $\operatorname{tr}\left(A_{i j}\right) \geq 1$. Moreover, if $\alpha_{m n-k}>0$, then $\operatorname{tr}\left(A_{i j}\right)>1$. On the other hand, we have

$$
m n=\operatorname{tr}\left(I_{m n}\right)=\operatorname{tr}\left(\phi\left(I_{m n}\right)\right)=\operatorname{tr}\left(\phi\left(\sum_{i, j} E_{i i} \otimes E_{j j}\right)\right)=\operatorname{tr}\left(\sum_{i, j} A_{i j}\right) \geq m n .
$$

This yields that $A_{i j}$ is a positive semidefinite matrix with trace one.

Similarly, if $m n<2 k$, then $\alpha_{m n-k+1}+\cdots+\alpha_{k} \geq 0$ and, thus, $\operatorname{tr}\left(A_{i j}\right) \leq 1$. Therefore,

$$
m n=\operatorname{tr}\left(I_{m n}\right)=\operatorname{tr}\left(\phi\left(I_{m n}\right)\right)=\operatorname{tr}\left(\phi\left(\sum_{i, j} E_{i i} \otimes E_{j j}\right)\right)=\operatorname{tr}\left(\sum_{i, j} A_{i j}\right) \leq m n,
$$

which yields that $A_{i j}$ is a positive semidefinite matrix with trace one.

We can apply the same argument to show that for any orthonormal bases $\left\{x_{1}, \ldots, x_{m}\right\} \subseteq \mathbb{C}^{m}$ and $\left\{y_{1}, \ldots, y_{n}\right\} \subseteq \mathbb{C}^{n}, \operatorname{tr}\left(\phi\left(x_{i} x_{i}^{*} \otimes y_{j} y_{j}^{*}\right)\right)=1$. Thus, $\phi$ is trace preserving for all Hermitian $A \otimes B$ and, hence, for all matrices in $M_{m n}$.

Lemma 2.4. Let $k \in\{2, \ldots, N-2\}$ and $X, Y \in H_{N}$ with $W_{k}(X)=W_{k}(Y)=[0,1 / k]$ and $W_{k}(X+Y)=[0,2 / k]$. If

$$
X-Y=\operatorname{Diag}(1-(k-1) a, a, \ldots, a,-1-(k-1) a)
$$

with $a \in[-1 / k, 1 / k]$, then

$$
X=\operatorname{Diag}(1-(k-1) d, d, \ldots, d,-(k-1) d) \quad \text { and } Y=\operatorname{Diag}(-(k-1) d, d, \ldots, d, 1-(k-1) d)
$$

with $d \in\{0,1 / k\}$ so that $X-Y=\operatorname{Diag}(1,0, \ldots, 0,-1)$.

Proof. Suppose $X$ has diagonal entries $x_{1}, \ldots, x_{N}$ and $Y$ has diagonal entries $y_{1}, \ldots, y_{N}$. Then for any $1=i_{1}<\ldots<i_{k} \leq N-1$, we have

$$
\sum_{t=1}^{k} x_{i_{t}} \leq 1, \quad \sum_{t=1}^{k} y_{i_{t}} \geq 0, \quad \sum_{t=1}^{k} x_{i_{t}}-\sum_{t=1}^{k} y_{i_{t}}=1,
$$

which imply $x_{i_{1}}+\cdots+x_{i_{k}}=1$ equal to the sum of the $k$ largest eigenvalues of $X$ and $y_{i_{1}}+\cdots+y_{i_{k}}=0$ equal to the sum of the $k$ smallest eigenvalues of $Y$. Thus, applying Lemma 2.1, $x_{1}$ is the largest eigenvalue of $X, x_{2}=\cdots=x_{N-1}$ are the second largest eigenvalue of $X, y_{1}$ is the smallest 
eigenvalue of $Y$, and $y_{2}=\cdots=y_{N-1}$ are the second smallest eigenvalue of $Y$. Moreover, $X$ and $Y$ have the form

$$
X=\operatorname{Diag}(1-(k-1) \tilde{x}, \tilde{x}, \ldots, \tilde{x},-(k-1) \tilde{x}), \quad Y=\operatorname{Diag}(-(k-1) \tilde{y}, \tilde{y}, \ldots, \tilde{y}, 1-(k-1) \tilde{y})
$$

with $\tilde{x}, \tilde{y} \in[0,1 / k]$. Hence, $X+Y=\operatorname{Diag}(1-(k-1) \tilde{x}-(k-1) \tilde{y}, \tilde{x}+\tilde{y}, \ldots, \tilde{x}+\tilde{y}, 1-(k-1) \tilde{x}-(k-1) \tilde{y})$ satisfies $W_{k}(X+Y)=[0,2 / k]$. So, either $($ a) $k(\tilde{x}+\tilde{y})=2$, which implies that $\tilde{x}=\tilde{y}=1 / k$, or (b) $k(\tilde{x}+\tilde{y})=0$, which implies that $\tilde{x}=\tilde{y}=0$.

Lemma 2.5. Let $2 \leq k \leq m n / 2$ be an integer and $\phi: M_{m n} \rightarrow M_{m n}$ be a linear map satisfying (6)). Then for any orthonormal bases $\left\{x_{1}, \ldots, x_{m}\right\} \subseteq \mathbb{C}^{m}$ and $\left\{y_{1}, \ldots, y_{n}\right\} \subseteq \mathbb{C}^{n}$, either

(1) there is a unitary $U \in M_{m n}$ such that $U^{*} \phi\left(x_{i} x_{i}^{*} \otimes y_{j} y_{j}^{*}\right) U=x_{i} x_{i}^{*} \otimes y_{j} y_{j}^{*}$ for all $i=1, \ldots, m$, and $j=1, \ldots, n$, or

(2) $m n=2 k$ and there is a unitary $U \in M_{m n}$ such that $U^{*} \phi\left(x_{i} x_{i}^{*} \otimes y_{j} y_{j}^{*}\right) U=I_{m n} / k-x_{i} x_{i}^{*} \otimes y_{j} y_{j}^{*}$ for $i=1, \ldots, m$, and $j=1, \ldots, n$.

The proof of this lemma is rather technical. We will present it in the last part of this paper.

Denote by $\sigma(X)$ the spectrum of $X \in M_{n}$. The following example is useful in our proof.

Example 2.6. Suppose $m, n \geq 3$. Let $A=X \oplus O_{m-3}$ and $B=X \oplus O_{n-3}$ with $X=\left[\begin{array}{lll}0 & 3 & 0 \\ 0 & 0 & 1 \\ 0 & 0 & 0\end{array}\right]$. Then $A \otimes B$ is unitarily similar to

$$
O_{m n-7} \oplus\left[\begin{array}{ll}
0 & 3 \\
0 & 0
\end{array}\right] \oplus\left[\begin{array}{ll}
0 & 3 \\
0 & 0
\end{array}\right] \oplus\left[\begin{array}{lll}
0 & 9 & 0 \\
0 & 0 & 1 \\
0 & 0 & 0
\end{array}\right]
$$

and $A \otimes B^{t}$ is unitarily similar to

$$
O_{m n-7} \oplus\left[\begin{array}{ll}
0 & 1 \\
0 & 0
\end{array}\right] \oplus\left[\begin{array}{ll}
0 & 9 \\
0 & 0
\end{array}\right] \oplus\left[\begin{array}{lll}
0 & 3 & 0 \\
0 & 0 & 3 \\
0 & 0 & 0
\end{array}\right] .
$$

Consequently,

$$
\begin{aligned}
\sigma\left(\left(A \otimes B+(A \otimes B)^{*}\right) / 2\right) & =\{-\sqrt{41 / 2},-3 / 2,-3 / 2,0, \ldots, 0,3 / 2,3 / 2, \sqrt{41 / 2}\}, \\
\sigma\left(\left(A \otimes B^{t}+\left(A \otimes B^{t}\right)^{*}\right) / 2\right) & =\{-9 / 2,-\sqrt{9 / 2},-1 / 2,0, \ldots, 0,1 / 2, \sqrt{9 / 2}, 9 / 2\} .
\end{aligned}
$$

Applying Proposition 1.2, one see that $\operatorname{Re}\left(W_{k}(A \otimes B)\right) \neq \operatorname{Re}\left(W_{k}\left(A \otimes B^{t}\right)\right)$, and hence $W_{k}(A \otimes B) \neq$ $W_{k}\left(A \otimes B^{t}\right)$ for any $k \in\{1, \ldots, m n-1\}$.

Now we are ready to present the proof of Theorem 1.1.

\section{Proof of Theorem 1.1}

Note that the 1-numerical range is just the classical numerical range. The case $k=1$ has been obtained in [6, Theorem 2.1]. Since $(n-k) W_{n-k}(A)=\operatorname{tr}(A)-k W_{k}(A)$, by Lemma 2.3, we have

$$
W_{k}(\phi(A))=W_{k}(A) \quad \Longleftrightarrow \quad W_{n-k}(\phi(A))=W_{n-k}(A) .
$$

Therefore, we can focus our proof on $2 \leq k \leq m n / 2$, with $m n \geq 4$. 
Note that $W_{k}(X)=W_{k}(\phi(X)), W_{k}(X)=W_{k}\left(X^{t}\right), W_{k}(X)=W_{k}\left(U^{*} X U\right)$ for any unitary $U$

and $X$ in $M_{m n}$. Furthermore, if $A \in M_{2}$, then $A=U_{A} A^{t} U_{A}$ for some unitary $U_{A}$ depending on $A$ so that for any $B \in M_{n}$, the matrix $A \otimes B$ is unitarily similar to $A^{t} \otimes B$. Thus, $W_{k}(A \otimes$ $B)=W_{k}\left(A^{t} \otimes B\right)=W_{k}\left(\left(A^{t} \otimes B\right)^{t}\right)=W_{k}\left(A \otimes B^{t}\right)$. Similarly, if $A \in M_{m}$ and $B \in M_{2}$, then $W_{k}(A \otimes B)=W_{k}\left(A \otimes B^{t}\right)=W_{k}\left(A^{t} \otimes B\right)$. Combining the above, we get the sufficiency.

For the converse, suppose $W_{k}(A \otimes B)=W_{k}(\phi(A \otimes B))$ for all $(A, B) \in M_{m} \times M_{n}$. Suppose $m n \neq 2 k$. Then by Lemma 2.5, (1) always holds. So, for any Hermitian $A \in M_{m}$ and $B \in M_{n}$ with spectral decomposition $A=\sum a_{i} x_{i} x_{i}^{*}$ and $B=\sum b_{j} y_{j} y_{j}^{*}$, we see that $\phi(A \otimes B)$ is unitarily similar to $A \otimes B$. So, $A \otimes B$ and $\phi(A \otimes B)$ always have the same eigenvalues. Thus, by [4, Theorem 3.2], there is a unitary $V$ such that $\phi$ has the form $A \otimes B \mapsto V^{*} \varphi(A \otimes B) V$ for any Hermitian $A \in M_{m}$ and $B \in M_{n}$, where $\varphi$ is one of the following forms:

(1) $A \otimes B \mapsto A \otimes B$,

(2) $A \otimes B \mapsto A \otimes B^{t}$,

(3) $A \otimes B \mapsto A^{t} \otimes B$,

(4) $A \otimes B \mapsto A^{t} \otimes B^{t}$.

By linearity, the map $\phi$ can only have one of these forms on $M_{m n}$. However, if $m, n \geq 3$, we see that $\varphi$ cannot be of the form (2) or (3) by Example 2.6. So, $\varphi$ can only be of the form (1) or (4). The desired conclusion holds.

Now, suppose $m n=2 k$. We claim that either (1) in Lemma 2.5 holds for any choice of orthonormal bases $\left\{x_{1}, \ldots, x_{m}\right\} \subseteq \mathbb{C}^{m}$ and $\left\{y_{1}, \ldots, y_{n}\right\} \subseteq \mathbb{C}^{n}$, or (2) in Lemma 2.5 holds for any choice of orthonormal bases $\left\{x_{1}, \ldots, x_{m}\right\} \subseteq \mathbb{C}^{m}$ and $\left\{y_{1}, \ldots, y_{n}\right\} \subseteq \mathbb{C}^{n}$. To see this, note that the set

$$
S=\left\{(x, y): x \in \mathbb{C}^{m}, y \in \mathbb{C}^{n}, x^{*} x=1=y^{*} y\right\}
$$

is path connected because the unit spheres in $\mathbb{C}^{m}$ and $\mathbb{C}^{n}$ are path connected. Consider the continuous map from $S$ to reals defined by $f(x, y) \mapsto\left|\operatorname{det}\left(\phi\left(x x^{*} \otimes y y^{*}\right)\right)\right|$. If (1) holds for a pair of orthonormal bases containing $x$ and $y$, then $f(x, y)=0$; if (2) holds for a pair of orthonormal bases containing $x$ and $y$, then $f(x, y)=\left|\operatorname{det}\left(I_{m n} / k-E_{11} \otimes E_{11}\right)\right|=(1 / k)^{m n-1}(1-1 / k)$. It follows that either $f(x, y)=0$ for all $(x, y) \in S$ so that (1) always holds, or $f(x, y)=(1 / k)^{m n}(1-1 / k)$ for all $(x, y) \in S$ so that (2) always holds.

If (1) holds for all orthonormal bases $\left\{x_{1}, \ldots, x_{m}\right\} \subseteq \mathbb{C}^{m}$ and $\left\{y_{1}, \ldots, y_{n}\right\} \subseteq \mathbb{C}^{n}$, then, by the argument in the case of $m n \neq 2 k$, we see that $\phi$ has the desired form. If (2) holds for all orthonormal bases $\left\{x_{1}, \ldots, x_{m}\right\} \subseteq \mathbb{C}^{m}$ and $\left\{y_{1}, \ldots, y_{n}\right\} \subseteq \mathbb{C}^{n}$, then compose $\phi$ with the map $X \mapsto(\operatorname{tr} X) I / k-X$ so that the resulting map satisfies (1). The result follows.

Finally, we give the proof of Lemma 2.5.

\section{Proof of Lemma 2.5.}

By Lemma 2.3 (a), $\phi$ maps Hermitian matrices to Hermitian matrices. We may focus on the case that $x_{i} x_{i}^{*}=E_{i i}$ for $i=1, \ldots, m$ and $y_{j} y_{j}^{*}=E_{j j}$ for $j=1, \ldots, n$. Otherwise, replace $\phi$ by the map $\tilde{\phi}(A \otimes B)=\phi\left(V_{1} A V_{1}^{*} \otimes V_{2} B V_{2}^{*}\right)$ so that $V_{1} \in M_{m}$ and $V_{2} \in M_{n}$ are unitary matrices satisfying $V_{1} x_{i} x_{i}^{*} V_{1}^{*}=E_{i i}$ for $i=1, \ldots, m$, and $V_{2} y_{j} y_{j}^{*} V_{2}^{*}=E_{j j}$ for $j=1, \ldots, n$. 
We divide the proof into three cases, namely
(a) $m n \neq 2 k$,
(b) $m n=2 k, m \leq 3$ and $n \leq 3$
and
(c) $m n=2 k, m \geq 4$ or $n \geq 4$.

2.1. The case $m n \neq 2 k$.

Claim 1. There exists a unitary $U \in M_{m n}$ such that

$$
\phi\left(E_{i i} \otimes E_{j j}\right)=U\left(E_{i i} \otimes E_{j j}\right) U^{*}
$$

for all $1 \leq i \leq m$ and $1 \leq j \leq n$.

It suffices to prove that

$$
\phi\left(E_{i i} \otimes E_{j j}\right) \perp \phi\left(E_{r r} \otimes E_{s s}\right)
$$

for all pairs $(i, j) \neq(r, s)$ with $1 \leq i, r \leq m$ and $1 \leq j, s \leq n$.

First, suppose that $i=r$ or $j=s$. Considering

$$
W_{k}\left(\phi\left(E_{i i} \otimes E_{j j}\right)-\phi\left(E_{r r} \otimes E_{s s}\right)\right)=W_{k}\left(E_{i i} \otimes E_{j j}-E_{r r} \otimes E_{s s}\right)=[-1 / k, 1 / k],
$$

applying Lemma 2.3 and Lemma 2.2, we conclude that (9) holds.

Now, let $i \neq r$ and $j \neq s$. We may assume that $2 \leq k \leq m n / 2 \leq m n-2$, we consider

$W_{k}\left(\phi\left(E_{i i} \otimes\left(E_{j j}+E_{s s}\right)\right)-\phi\left(E_{r r} \otimes\left(E_{j j}+E_{s s}\right)\right)\right)=W_{k}\left(E_{i i} \otimes\left(E_{j j}+E_{s s}\right)-E_{r r} \otimes\left(E_{j j}+E_{s s}\right)\right)=[-2 / k, 2 / k]$. Applying Lemma 2.2 again, it follows that $\phi\left(E_{i i} \otimes\left(E_{j j}+E_{s s}\right)\right) \perp \phi\left(E_{r r} \otimes\left(E_{j j}+E_{s s}\right)\right)$. Hence, we have (9).

\subsection{The case $m n=2 k, m \leq 3$ and $n \leq 3$.}

Since $m n=2 k$ is an even integer, without loss of generality we may assume that $n$ is even. So it suffices to consider the cases when $n=2$ and $m \in\{2,3\}$.

Claim 2. Let $m \in\{2,3\}$ and $A_{i}=\phi\left(E_{i i} \otimes\left(E_{11}-E_{22}\right)\right) \in M_{m} \otimes M_{2}$ for $i=1, \ldots, m$. Then there is a unitary $U \in M_{2 m}$ such that $\phi\left(A_{i}\right)=U\left(E_{i i} \otimes\left(E_{11}-E_{22}\right)\right) U^{*}$ for $i=1, \ldots, m$.

We only need to show that $A_{1}, \ldots, A_{m}$ are mutually orthogonal and each $A_{i}$ has eigenvalues $1,-1,0, \ldots, 0$. Note that $W_{k}\left(A_{i}\right)=[-1 / k, 1 / k]$ for $i=1, \ldots, m$. So $\lambda_{1}\left(A_{i}\right)+\cdots+\lambda_{k}\left(A_{i}\right)=1$ and $\lambda_{k+1}\left(A_{i}\right)+\cdots+\lambda_{2 m}\left(A_{i}\right)=-1$. Since $W_{k}\left(A_{1}+A_{2}\right)=[-2 / k, 2 / k]$, we see that

$$
\sum_{j=1}^{k} \lambda_{j}\left(A_{1}+A_{2}\right)=\sum_{j=1}^{k}\left(\lambda_{j}\left(A_{1}\right)+\lambda_{j}\left(A_{2}\right)\right)=2
$$

and

$$
\sum_{j=k+1}^{2 m} \lambda_{j}\left(A_{1}+A_{2}\right)=\sum_{j=k+1}^{2 m}\left(\lambda_{j}\left(A_{1}\right)+\lambda_{j}\left(A_{2}\right)\right)=-2 .
$$

So, by a unitary similarity and applying Lemma 2.1, we may assume that $A_{1}=B_{1} \oplus C_{1}$ and $A_{2}=$ $B_{2} \oplus C_{2}$ so that $B_{i}$ has eigenvalues $\lambda_{1}\left(A_{i}\right), \ldots, \lambda_{k}\left(A_{i}\right)$ and $C_{i}$ has eigenvalues $\lambda_{k+1}\left(A_{i}\right), \ldots, \lambda_{2 m}\left(A_{i}\right)$, $i=1,2$. As $W_{k}\left(A_{1}-A_{2}\right)=[-2 / k, 2 / k]$, we see that $\left(B_{1}-B_{2}\right) \oplus\left(C_{1}-C_{2}\right)$ has eigenvalues $\gamma_{1} \geq \cdots \geq \gamma_{2 m}$ such that $\gamma_{1}+\cdots+\gamma_{k}=2$ and $\gamma_{k+1}+\cdots+\gamma_{2 m}=-2$. Clearly, $\gamma_{1}, \ldots, \gamma_{k}$ cannot 
all come from $B_{1}-B_{2}$, else, $\gamma_{1}+\cdots+\gamma_{k}=\operatorname{tr}\left(B_{1}-B_{2}\right)=0$. Similarly, $\gamma_{1}, \ldots, \gamma_{k}$ cannot all come from $C_{1}-C_{2}$. Now we distinguish two cases.

Case 1. $m=2$. In this case we see that an eigenvalue of $B_{1}-B_{2}$ and an eigenvalue of $C_{1}-C_{2}$ sum up to $\gamma_{1}+\gamma_{2}=2$. Since $\lambda_{1}\left(B_{1}-B_{2}\right) \leq \lambda_{1}\left(A_{1}\right)-\lambda_{2}\left(A_{2}\right)$ and

$$
\lambda_{1}\left(C_{1}-C_{2}\right) \leq \lambda_{3}\left(A_{1}\right)-\lambda_{4}\left(A_{2}\right),
$$

we have

$$
\begin{aligned}
2 & =\gamma_{1}+\gamma_{2} \leq \lambda_{1}\left(A_{1}\right)+\lambda_{3}\left(A_{1}\right)-\lambda_{2}\left(A_{2}\right)-\lambda_{4}\left(A_{2}\right) \\
& \leq \lambda_{1}\left(A_{1}\right)+\lambda_{2}\left(A_{1}\right)-\lambda_{3}\left(A_{2}\right)-\lambda_{4}\left(A_{2}\right)=2 .
\end{aligned}
$$

It follows that

$$
\lambda_{2}\left(A_{1}\right)=\lambda_{3}\left(A_{1}\right) \quad \text { and } \quad \lambda_{2}\left(A_{2}\right)=\lambda_{3}\left(A_{2}\right) .
$$

Without loss of generality, assume that $A_{1}$ is unitarily similar to a matrix of the form

$$
\operatorname{Diag}(1-a, a, a,-1-a) \quad \text { with } \quad a \in[-1 / 2,1 / 2] .
$$

By Lemma 2.4, we conclude that $a=0$.

Similarly, we can show that $A_{2}$ has eigenvalues $1,-1,0,0$. It is then easy to show that $A_{1}, A_{2}$ are orthogonal.

Case 2. $m=3$. We have two subcases.

Subcase 2.1. An eigenvalue of $B_{1}-B_{2}$ and two eigenvalues of $C_{1}-C_{2}$ sum up to $\gamma_{1}+\gamma_{2}+\gamma_{3}=2$.

Since $\lambda_{1}\left(B_{1}-B_{2}\right) \leq \lambda_{1}\left(A_{1}\right)-\lambda_{3}\left(A_{2}\right)$ and

$$
\begin{aligned}
\lambda_{1}\left(C_{1}-C_{2}\right)+\lambda_{2}\left(C_{1}-C_{2}\right) & \leq \lambda_{1}\left(C_{1}\right)+\lambda_{2}\left(C_{1}\right)-\lambda_{2}\left(C_{2}\right)-\lambda_{3}\left(C_{2}\right) \\
& =\lambda_{4}\left(A_{1}\right)+\lambda_{5}\left(A_{1}\right)-\lambda_{5}\left(A_{2}\right)-\lambda_{6}\left(A_{2}\right),
\end{aligned}
$$

we have

$$
\begin{aligned}
2 & =\gamma_{1}+\gamma_{2}+\gamma_{3} \leq \lambda_{1}\left(A_{1}\right)+\lambda_{4}\left(A_{1}\right)+\lambda_{5}\left(A_{1}\right)-\lambda_{3}\left(A_{2}\right)-\lambda_{5}\left(A_{2}\right)-\lambda_{6}\left(A_{2}\right) \\
& \leq \lambda_{1}\left(A_{1}\right)+\lambda_{2}\left(A_{1}\right)+\lambda_{3}\left(A_{1}\right)-\lambda_{4}\left(A_{2}\right)-\lambda_{5}\left(A_{2}\right)-\lambda_{6}\left(A_{2}\right)=2 .
\end{aligned}
$$

It follows that

$$
\lambda_{2}\left(A_{1}\right)=\cdots=\lambda_{5}\left(A_{1}\right) \quad \text { and } \quad \lambda_{3}\left(A_{2}\right)=\lambda_{4}\left(A_{2}\right) .
$$

Subcase 2.2. An eigenvalue of $C_{1}-C_{2}$ and two eigenvalues of $B_{1}-B_{2}$ sum up to $\gamma_{1}+\gamma_{2}+\gamma_{3}=2$. Then

$$
\lambda_{3}\left(A_{1}\right)=\lambda_{4}\left(A_{1}\right) \quad \text { and } \quad \lambda_{2}\left(A_{2}\right)=\cdots=\lambda_{5}\left(A_{2}\right) .
$$

Without loss of generality, assume that $A_{1}$ is unitarily similar to a matrix of the form

$$
\operatorname{Diag}(1-2 a, a, a, a, a,-1-2 a) \quad \text { with } \quad a \in[-1 / 3,1 / 3] .
$$

By Lemma 2.4, we conclude that $a=0$. 
Now, applying the arguments to $A_{2}$ and $A_{3}$, we conclude that one of the matrices $A_{2}$ and $A_{3}$, say, $A_{2}$, has eigenvalues $1,-1,0,0,0,0$. Then it follows from Lemma 2.2 that $A_{1}$ and $A_{2}$ are orthogonal. So, we may assume that $A_{1}=\operatorname{Diag}(1,0,0,-1,0,0)$ and $A_{2}=\operatorname{Diag}(0,1,0,0,-1,0)$. Note that

$$
W_{3}\left(A_{1}+A_{2}-A_{3}\right)=W_{3}\left(A_{1}+A_{2}+A_{3}\right)=[-1,1] .
$$

We see that $A_{3}=B_{3} \oplus C_{3}$ such that $B_{3}$ has eigenvalues $1-2 c, c, c$ and $C_{3}$ has eigenvalues $c, c,-1-2 c$. Now, applying Lemma 2.4 on $A_{3}$, we conclude that $A_{3}$ also has eigenvalues $1,-1,0,0,0,0$. It follows from Lemma 2.2 that $A_{1}, A_{2}, A_{3}$ are mutually orthogonal. Thus, we obtain the claim.

Using the notation as in Claim 2, we see that there is a unitary $U \in M_{2 m}$ such that $A_{i}=$ $U\left(E_{i i} \otimes\left(E_{11}-E_{22}\right)\right) U^{*}$. By Lemma 2.4, for each $i=1, \ldots, m$,

$$
\phi\left(E_{i i} \otimes E_{j j}\right)=U\left(E_{i i} \otimes E_{j j}\right) U^{*}, \quad j=1,2,
$$

or

$$
\phi\left(E_{i i} \otimes E_{j j}\right)=I_{2 m} / k-P_{i}\left(E_{i i} \otimes E_{j j}\right) P_{i}^{t}, \quad j=1,2,
$$

for a suitable permutation matrix $P_{i} \in M_{2 m}$. Because $\sum_{i, j} \phi\left(E_{i i} \otimes E_{j j}\right)=I_{2 m}$, either (10) holds for all $i=1, \ldots, m$, or (11) holds for all $i=1, \ldots, m$. In the latter case, the map $\tilde{\phi}(A)=$ $\operatorname{tr}(A) / k I_{m n}-\phi(A)$ must satisfy (10). This shows that $P_{1}=\cdots=P_{m}$.

\subsection{The case $m n=2 k, m \geq 4$ or $n \geq 4$.}

Without loss of generality, we assume $m \geq 4$. If $A_{i j}=\phi\left(E_{i i} \otimes E_{j j}\right)$ is positive semidefinite for any $1 \leq i \leq m$ and $1 \leq j \leq n$, then applying the same arguments as in the previous case, we conclude that $\phi$ satisfies (1). Now suppose there exist some $i_{0}$ and $j_{0}$ such that $A_{i_{0} j_{0}}$ has negative eigenvalues. Without loss of generality, we assume $i_{0}=j_{0}=1$.

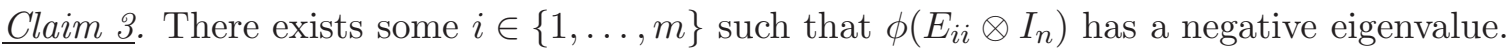

For $1 \leq i \leq m$, we denote the eigenvalues of $\phi\left(E_{i i} \otimes I_{n}\right)$ by $a_{1}(i) \geq a_{2}(i) \geq \cdots \geq a_{m n}(i)$. Since $W_{k}\left(\phi\left(E_{i i} \otimes I_{n}\right)\right)=[0, n / k]$, we have $\sum_{j=1}^{k} a_{j}(i)=n$ and $\sum_{j=k+1}^{m n} a_{j}(i)=0$ for $1 \leq i \leq m$. Suppose $\phi\left(E_{i i} \otimes I_{n}\right) \geq 0$ for all $1 \leq i \leq m$. Since $W_{k}\left(\phi\left(E_{11}+E_{22}\right) \otimes I_{n}\right)=[0,2 n / k]$, without loss of generality we can assume $\phi\left(\left(E_{11}+E_{22}\right) \otimes I_{n}\right)=\operatorname{Diag}\left(r_{1}, \ldots, r_{m n}\right)$ with $r_{1} \geq r_{2} \geq \cdots \geq r_{k} \geq r_{k+1}=\cdots=$ $r_{m n}=0$. Let $\phi\left(E_{11} \otimes I_{n}\right)=\left(x_{i j}\right)$ and $\phi\left(E_{22} \otimes I_{n}\right)=\left(y_{i j}\right)$. Then

$$
\sum_{i=1}^{k} x_{i i}=\sum_{i=1}^{k} y_{i i}=n=\sum_{i=1}^{k} a_{i}(1)=\sum_{i=1}^{k} a_{i}(2) \text {. }
$$

By Lemma 2.1. $\phi\left(E_{11} \otimes I_{n}\right)=X \oplus 0_{k}$ and $\phi\left(E_{22} \otimes I_{n}\right)=Y \oplus 0_{k}$ with $\operatorname{tr} X=\operatorname{tr} Y=n$. Moreover, $W_{k}\left(\phi\left(\left(E_{11}-E_{22}\right) \otimes I_{n}\right)\right)=[-n / k, n / k]$. Thus, applying Lemma 2.2 we have $X \perp Y$. It follows that $X$ is singular and $a_{k}(1)=a_{k+1}(1)=\cdots=a_{m n}(1)=0$. Suppose $V \in M_{m n}$ is a unitary matrix such that

$$
\operatorname{Diag}\left(a_{1}(1), \ldots, a_{k-1}(1), 0, \ldots, 0\right)=V \phi\left(E_{11} \otimes I_{n}\right) V^{*}=\sum_{j=1}^{n} V \phi\left(E_{11} \otimes E_{j j}\right) V^{*}
$$


Denote the diagonal entries of $V \phi\left(E_{11} \otimes E_{j j}\right) V^{*}$ by $d_{1}(j), \ldots, d_{m n}(j)$. Then

$$
n=\sum_{i=1}^{k-1} a_{i}(1)+a_{s}(1)=\sum_{j=1}^{n}\left(\sum_{i=1}^{k-1} d_{i}(j)+d_{s}(j)\right)
$$

for every $s \in\{k, \ldots, m n\}$ and $W_{k}\left(\phi\left(E_{11} \otimes E_{j j}\right)\right)=[0,1 / k]$ ensures that $\sum_{i=1}^{k-1} d_{i}(j)+d_{s}(j)=1$ for every $s \in\{k, \ldots, m n\}$. Applying Lemma 2.1 again, we see that for every $j \in\{1, \ldots, n\}$, we have

$$
V \phi\left(E_{11} \otimes E_{j j}\right) V^{*}=R_{j} \oplus t_{j} I_{k+1},
$$

where each eigenvalue of $R_{j}$ is larger than or equal to $t_{j}$. Further, $0 \in W_{k}\left(\phi\left(E_{11} \otimes E_{j j}\right)\right)$ implies $t_{j}=0$ for $1 \leq j \leq n$, which contradicts with the assumption that $\phi\left(E_{11} \otimes E_{11}\right)$ is not positive semidefinite.

Claim 4. Suppose there exists $i \in\{1, \ldots, m\}$ such that the eigenvalues of $\phi\left(E_{i i} \otimes I_{n}\right)$ are $a_{1}(i) \geq$ $a_{2}(i) \geq \cdots \geq a_{m n}(i)$ with $a_{m n}(i)<0$. Then

$$
a_{1}(i)=a_{2}(i)=\cdots=a_{k+1}(i) .
$$

Moreover, $\phi\left(E_{j j} \otimes I_{n}\right)$ has a negative eigenvalue for every $j \in\{1, \ldots, m\}$.

Without loss of generality, we assume $i=1$ and

$$
\phi\left(E_{11} \otimes I_{n}\right)=\operatorname{Diag}\left(a_{1}(1), \cdots, a_{m n}(1)\right)
$$

with

$$
a_{1}(1) \geq a_{2}(1) \geq \cdots \geq a_{m n}(1)
$$

where $a_{1}(1), \ldots, a_{k+1}(1)$ are not identical. Let us denote the diagonal entries of $\phi\left(E_{j j} \otimes I_{n}\right)$ by $h_{1}(j), \ldots, h_{m n}(j)$ and the diagonal entries of $U \phi\left(E_{j j} \otimes I_{n}\right) U^{*}$ by $h_{1}(U, j), \ldots, h_{m n}(U, j)$. Note that $a_{k}(1)$ and $a_{k+1}(1)$ must be equal. Otherwise, by the fact that $W_{k}\left(\phi\left(\left(E_{11}+E_{j j}\right) \otimes I_{n}\right)\right)=[0,2 n / k]$, we have $\sum_{r=1}^{k} a_{r}(1)=\sum_{r=1}^{k} h_{r}(j)=n$ for $j=2, \ldots, m$. But then, if $Z=\sum_{j=1}^{m} \phi\left(E_{j j} \otimes I_{n}\right)$, the leading $k \times k$ submatrix of $Z$ will have trace $m n$, which contradicts with $W_{k}\left(\phi\left(I_{m n}\right)\right)=\{1\}$. Suppose $a_{1}(1)>a_{k}(1)$, i.e., there are integers $s, t \in\{1, \ldots, k-1\}$ such that

$$
a_{1}(1) \geq \cdots \geq a_{s}(1)>a_{s+1}(1)=\cdots=a_{k+t}(1)>a_{k+t+1}(1) \geq \cdots \geq a_{m n}(1) .
$$

We are going to show that

$$
h_{1}(j)=\cdots=h_{s}(j)=h_{k+t+1}(j)=\cdots=h_{m n}(j) \quad \text { for } j=2, \ldots, m .
$$

Let $\gamma=m / 2$ when $m$ is even and $\gamma=(m+1) / 2$ when $m$ is odd. Denote by

$$
\begin{gathered}
G=\phi\left(\left(2\left(E_{11}+\cdots+E_{\gamma-1, \gamma-1}\right)+E_{\gamma, \gamma}\right) \otimes I_{n}\right), \\
G_{1}=\phi\left(\left(E_{11}+\cdots+E_{\gamma-1, \gamma-1}\right) \otimes I_{n}\right), \quad \text { and } \quad G_{2}=\phi\left(\left(E_{11}+\cdots+E_{\gamma, \gamma}\right) \otimes I_{n}\right) .
\end{gathered}
$$

Then we have

$$
W_{k}\left(G_{1}\right)=[0,(\gamma-1) n / k], \quad w_{k}(G)=w_{k}\left(G_{1}\right)+w_{k}\left(G_{2}\right),
$$

where the $k$-numerical radius $w_{k}(G), w_{k}\left(G_{1}\right)$, and $w_{k}\left(G_{2}\right)$ are the right end points of $W_{k}(G), W_{k}\left(G_{1}\right)$, and $W_{k}\left(G_{2}\right)$, respectively. Let $U$ be a unitary such that the sum of the first $k$ diagonal entries of 
$U G U^{*}$ equals to $k w_{k}(G)$. Then the sum of the first $k$ diagonal entries of $U G_{i} U^{*}$ equals to $k w_{k}\left(G_{i}\right)$ for $i=1,2$. We assert that the following conditions hold.

(a) $\sum_{p=1}^{k} h_{p}(U, \gamma)=n$ when $m$ is even and $\sum_{p=1}^{k} h_{p}(U, \gamma)=n / 2$ when $m$ is odd.

(b) $U \phi\left(E_{j j} \otimes I_{n}\right) U^{*}=B_{j 1} \oplus B_{j 2}$ with $B_{j 1} \in M_{k}$ and $\operatorname{tr}\left(B_{j 1}\right)=n$ for $j=1, \ldots, \gamma-1$.

(c) $U \phi\left(E_{j j} \otimes I_{n}\right) U^{*}=B_{j 1} \oplus B_{j 2}$ with $B_{j 1} \in M_{k}$ and $\operatorname{tr}\left(B_{j 2}\right)=n$ for $j=\gamma+1, \ldots, m$.

Since

$$
\begin{aligned}
(\gamma-1) n & =k w_{k}\left(G_{1}\right)=\sum_{j=1}^{\gamma-1} \sum_{r=1}^{k} h_{r}(U, j) \leq \sum_{j=1}^{\gamma-1} n=(\gamma-1) n, \text { and } \\
\frac{m n}{2} & =k w_{k}\left(G_{2}\right)=\sum_{j=1}^{\gamma} \sum_{r=1}^{k} h_{r}(U, j) \leq \frac{m n}{2}
\end{aligned}
$$

It follows that $\sum_{p=1}^{k} h_{p}(U, j)=n$ for $j=1, \ldots, \gamma-1$ and (a) holds. Applying Lemma 2.1, we have the condition (b).

For any $j \in\{\gamma+1, \ldots, m\}$, since

$$
W_{k}\left(\phi\left(E_{j j} \otimes I_{n}\right)\right)=W_{k}\left(E_{j j} \otimes I_{n}\right)=[0, n / k],
$$

the sum of any $k$ diagonal entries of $U \phi\left(E_{j j} \otimes I_{n}\right) U^{*}$ lies in $[0, n]$. Now, the right end point of the set

$$
W_{k}\left(\phi\left(\left(E_{11}+\cdots+E_{\gamma, \gamma}+E_{j j}\right) \otimes I_{n}\right)\right)
$$

is 1 , and the sum of the first $k$ diagonal entries of $U \phi\left(\left(E_{11}+\cdots+E_{\gamma, \gamma}\right) \otimes I_{n}\right) U^{*}$ is $k$. We see that $\sum_{p=1}^{k} h_{p}(U, j)=0$ and $\sum_{p=k+1}^{m n} h_{p}(U, j)=n$. Hence, we get (c).

Suppose $U_{1}$ and $U_{2}$ are unitary matrices such that

$$
U_{1} B_{11} U_{1}^{*}=\operatorname{Diag}\left(a_{1}(1), \ldots, a_{k}(1)\right) \text { and } U_{2} B_{12} U_{2}^{*}=\operatorname{Diag}\left(a_{k+1}(1), \ldots, a_{m n}(1)\right) .
$$

Replace $U$ with $\left(U_{1} \oplus U_{2}\right) U$. Then the new matrix $U$ also satisfies (a), (b) and (c). Moreover, $U$ is of diagonal block form $U_{3} \oplus U_{4} \oplus U_{5}$ with $U_{3} \in M_{s}, U_{5} \in M_{k-t}$. Since any unitary $U_{3}$ and $U_{5}$ will yield the same summation of the first $k$ diagonal entries of $U G U^{*}$, we can assume $U=I_{s} \oplus U_{4} \oplus I_{k-t}$. Thus, for $j=\gamma+1, \ldots, m$, it follows from (c) that

$$
h_{p}(j)=h_{p}(U, j) \leq h_{q}(U, j)=h_{q}(j) \quad \text { for all } p \in\{1, \ldots, s\} \text { and } q \in\{k+t+1, \ldots, m n\} .
$$

On the other hand, since $W_{k}\left(\phi\left(\left(E_{11}+E_{j j}\right) \otimes I_{n}\right)\right)=[0,2 n / k]$, there exists a unitary matrix $V$ of the form $V=I_{s} \oplus V_{1} \oplus I_{k-t}$ such that $\sum_{p=1}^{k} h_{p}(V, j)=n$, which implies

$$
h_{p}(j) \geq h_{q}(j) \quad \text { for all } p \in\{1, \ldots, s\} \text { and } q \in\{k+t+1, \ldots, m n\} .
$$

Hence, we have

$$
h_{1}(j)=\cdots=h_{s}(j)=h_{k+t+1}(j)=\cdots=h_{m n}(j) \quad \text { for } j=\gamma+1, \ldots, m .
$$

Interchanging the roles of $\{2, \ldots, \gamma\}$ and $\{\gamma+1, \ldots, 2 \gamma-1\}$ and applying the same argument, we have (15).

Let $T=\{1, k+1, \ldots, m n-1\}$. Note that $h_{1}(U, 1)=a_{1}(1)>a_{m n}(1)=h_{m n}(U, 1)$. We have

$$
\sum_{p \in T} h_{p}(U, 1)>\sum_{p=k+1}^{m n} h_{p}(U, 1)=0 .
$$


By the fact that $h_{1}(U, j)=h_{1}(j)=h_{m n}(j)=h_{m n}(U, j)$ for $j=2, \ldots, m$, we have

$$
\begin{aligned}
\sum_{p \in T}\left(h_{p}(U, 1)+\sum_{j=\gamma}^{m} h_{p}(U, j)\right) & =\sum_{p \in T} h_{p}(U, 1)+\sum_{j=\gamma}^{m}\left(\sum_{p=k+1}^{m n} h_{p}(U, j)\right) \\
& =\sum_{p \in T} h_{p}(U, 1)+k>k
\end{aligned}
$$

which contradicts with $w_{k}\left(\phi\left(\left(E_{11}+E_{\gamma, \gamma}+\cdots+E_{m m}\right) \otimes I_{n}\right)\right)=1$. Hence, we get (12).

Next, suppose there is some $2 \leq j \leq m$ such that $\phi\left(E_{j j} \otimes I_{n}\right) \geq 0$, say, $j=2$. Again, we can assume $\phi\left(E_{11} \otimes I_{n}\right)=\operatorname{Diag}\left(a_{1}(1), \ldots, a_{m n}(1)\right)$ with

$$
a_{1}(1)=\cdots=a_{k+t}(1)>a_{k+t+1}(1) \geq \cdots \geq a_{m n}(1), \quad 1 \leq t \leq k-1
$$

and $\phi\left(E_{22} \otimes I_{n}\right)=\operatorname{Diag}\left(a_{1}(2), \ldots, a_{m n}(2)\right)$ with

$$
a_{1}(2) \geq \cdots \geq a_{s}(2)>a_{s+1}(2)=\cdots=a_{m n}(2)=0, \quad 1 \leq s \leq k .
$$

Recall that there is a unitary matrix $U$ satisfying (a), (b), and (c). Suppose $U_{1}, U_{2}$ are unitary matrices such that $U_{1} B_{21} U_{1}^{*}=\operatorname{Diag}\left(a_{1}(2), \ldots, a_{k}(2)\right)$ and $U_{2} B_{12} U_{2}^{*}=\operatorname{Diag}\left(a_{k+1}(1), \ldots, a_{m n}(1)\right)$. Replace $U$ with $\left(U_{1} \oplus U_{2}\right) U$. Then the new matrix $U$ also satisfies (a), (b), and (c). Moreover,

$$
\begin{aligned}
& U \phi\left(E_{11} \otimes I_{n}\right) U^{*}=\operatorname{Diag}\left(a_{1}(1), \ldots, a_{m n}(1)\right), \\
& U \phi\left(E_{22} \otimes I_{n}\right) U^{*}=\operatorname{Diag}\left(a_{1}(2), \ldots, a_{m n}(2)\right)
\end{aligned}
$$

which implies that $U$ is of the form $U=U_{3} \oplus U_{4} \oplus U_{5}$ with $U_{3} \in M_{s}, U_{5} \in M_{k-t}$. We can assume $U_{3}=I_{s}$ and $U_{5}=I_{k-t}$.

For any given $j \in\{\gamma+1, \ldots, m\}$, we denote by $\alpha_{1} \geq \cdots \geq \alpha_{k}$ the eigenvalues of $B_{j 1}$ and $\beta_{1} \geq \cdots \geq \beta_{k}$ the eigenvalues of $B_{j 2}$. Then $\beta_{k} \geq \alpha_{1}$. By $W_{k}\left(\phi\left(\left(E_{22}+E_{j j}\right) \otimes I_{n}\right)\right)=[0,2 n / k]$, there is a unitary $V$ such that

$$
V\left(B_{21} \oplus B_{22}\right) V^{*}=Y \oplus 0 \text { and } V\left(B_{j 1} \oplus B_{j 2}\right) V^{*}=Z_{1} \oplus Z_{2}
$$

with $Y, Z_{1} \in M_{k}, \operatorname{tr}(Y)=\operatorname{tr}\left(Z_{1}\right)=n$. Suppose $W$ is a unitary matrix such that $W Y W^{*}=B_{21}$. Replace $V$ with $\left(W \oplus I_{k}\right) V$. Then we still have (18) with $Y=B_{21}$. Moreover, $V$ is of the form $V=V_{1} \oplus V_{2}$ with $V_{1} \in M_{s}$ and we can assume $V_{1}=I_{s}$. Partition $B_{j 1}$ and $Z_{1}$ as

$$
B_{j 1}=\left[\begin{array}{ll}
C_{11} & C_{12} \\
C_{21} & C_{22}
\end{array}\right], \quad Z_{1}=\left[\begin{array}{cc}
D_{11} & D_{12} \\
D_{21} & D_{22}
\end{array}\right]
$$

with $C_{11}, D_{11} \in M_{s}$. We can rewrite the second equation in (18) as

$$
\left[\begin{array}{ll}
I_{s} & \\
& V_{2}
\end{array}\right]\left[\begin{array}{lll}
C_{11} & C_{12} & \\
C_{21} & C_{22} & \\
& & B_{j 2}
\end{array}\right]\left[\begin{array}{ll}
I_{s} & \\
& V_{2}
\end{array}\right]^{*}=\left[\begin{array}{lll}
D_{11} & D_{12} & \\
D_{21} & D_{22} & \\
& & Z_{2}
\end{array}\right] .
$$

It is clear that $D_{11}=C_{11}$. Since $\operatorname{tr}\left(C_{11}+D_{22}\right)=n$ equals to the sum of the $k$ largest eigenvalues of $B_{j 1} \oplus B_{j 2}$, we see that $\operatorname{tr}\left(D_{22}\right)=\sum_{p=1}^{k-s} \beta_{p}$. Applying Lemma 2.1, we have $D_{12}=D_{21}^{*}=0$, which implies $C_{12}=C_{21}^{*}=0$ and $\sigma\left(C_{11}\right)=\left\{\alpha_{1} \ldots, \alpha_{s}\right\}$. It follows that $\alpha_{1}=\cdots=\alpha_{s}=\beta_{k-s+1}=\cdots=$ $\beta_{k}$ and $C_{11}=\alpha_{1} I_{s}$.

Similarly, considering $W_{k}\left(\phi\left(\left(E_{11}+E_{j j}\right) \otimes I_{n}\right)\right)=[0,2 n / k]$, there is a unitary $\tilde{V}$ such that

$$
\tilde{V}\left(B_{11} \oplus B_{12}\right) \tilde{V}^{*}=a_{1}(1) I_{k} \oplus \tilde{Y} \text { and } \tilde{V}\left(B_{j 1} \oplus B_{j 2}\right) \tilde{V}^{*}=\tilde{Z}_{1} \oplus \tilde{Z}_{2}
$$


with $\tilde{Z}_{1} \in M_{k}, \operatorname{tr}\left(\tilde{Z}_{1}\right)=n$. Suppose $\tilde{W}$ is a unitary matrix such that $\tilde{W} \tilde{Y} \tilde{W}^{*}=B_{12}$. Replace $\tilde{V}$ with $\left(I_{k} \oplus \tilde{W}\right) \tilde{V}$. Then we still have (19) with $\tilde{Y}=B_{12}$. Moreover, $\tilde{V}$ is of the form $\tilde{V}=\tilde{V}_{1} \oplus \tilde{V}_{2}$ with $V_{2} \in M_{k-t}$ and we can assume $V_{2}=I_{k-t}$. Partition $B_{j 2}$ and $\tilde{Z}_{2}$ as

$$
B_{j 2}=\left[\begin{array}{ll}
R_{11} & R_{12} \\
R_{21} & R_{22}
\end{array}\right], \quad \tilde{Z}_{2}=\left[\begin{array}{ll}
S_{11} & S_{12} \\
S_{21} & S_{22}
\end{array}\right]
$$

with $R_{11}, S_{11} \in M_{t}$. We can rewrite the second equation in (19) as

$$
\left[\begin{array}{cc}
\tilde{V}_{1} & \\
& I_{k-t}
\end{array}\right]\left[\begin{array}{llll}
C_{11} & & & \\
& C_{22} & & \\
& & R_{11} & R_{12} \\
& & R_{21} & R_{22}
\end{array}\right]\left[\begin{array}{ll}
\tilde{V}_{1} & \\
& I_{k-t}
\end{array}\right]^{*}=\left[\begin{array}{lll}
\tilde{Z}_{1} & & \\
& S_{11} & S_{12} \\
& S_{21} & S_{22}
\end{array}\right] .
$$

Since $\operatorname{tr}\left(\tilde{Z}_{1}\right)=n$ is the sum of the $k$ largest eigenvalues of $B_{j 1} \oplus B_{j 2}$, which equals to the sum of the $k$ largest eigenvalues of $C_{11} \oplus C_{22} \oplus R_{11}$, we see that the eigenvalues of $R_{11}$ are also the $t$ largest eigenvalues of $B_{j 2}$. Hence, we have $R_{12}=R_{21}^{*}=0$ and $R_{22}=\beta_{k} I_{k-t}=\alpha_{1} I_{k-t}$.

So we have

$$
h_{1}(j)=h_{1}(U, j)=h_{m n}(U, j)=h_{m n}(j) \text { for } j=\gamma+1, \ldots, m .
$$

Similarly, (20) holds for $j=2, \ldots, \gamma$.

Again, we have (17), which contradicts with $w_{k}\left(\phi\left(\left(E_{11}+E_{\gamma, \gamma}+\cdots+E_{m m}\right) \otimes I_{n}\right)\right)=1$. Therefore, $\phi\left(E_{j j} \otimes I_{n}\right)$ has a negative eigenvalue for every $1 \leq j \leq m$.

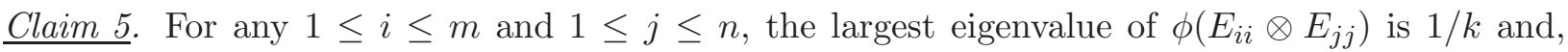
hence, $\frac{1}{k} I_{m n}-\phi\left(E_{i i} \otimes E_{j j}\right) \geq 0$.

Given any $1 \leq i \leq m$, by the previous claims, we can assume

$$
\phi\left(E_{i i} \otimes I_{n}\right)=\operatorname{Diag}\left(a_{1}(i), \ldots, a_{m n}(i)\right)
$$

with $a_{1}(i)=\cdots=a_{k+1}(i) \geq \cdots \geq a_{m n}(i)$ and $a_{m n}(i)<0$. Denote by $d_{1}(i, j), \ldots, d_{m n}(i, j)$ the diagonal entries of $\phi\left(E_{i i} \otimes E_{j j}\right)$. Then

$$
\sum_{u \in T} d_{u}(i, 1)=\cdots=\sum_{u \in T} d_{u}(i, n)=1
$$

for any $T \subseteq\{1, \ldots, k+1\}$ with $|T|=k$. It follows that $d_{u}(i, j)=1 / k$ for all $1 \leq u \leq k+1$ and $1 \leq j \leq n$.

Applying Lemma 2.1, each $\phi\left(E_{i i} \otimes E_{j j}\right)$ is of the form

$$
\operatorname{Diag}\left(d_{1}(i, j), \ldots, d_{k+1}(i, j)\right) \oplus X(i, j),
$$

where the largest eigenvalue of $X(i, j)$ is less than or equal to $1 / k$. Thus, we get the claim.

Now, let $\psi(A \otimes B)=(\operatorname{tr}(A \otimes B) / k) I_{m n}-\phi(A \otimes B)$. Then $W_{k}(\psi(A \otimes B))=W_{k}(A \otimes B)$ for all $A \in H_{m}, B \in H_{n}$, and $\psi\left(E_{i i} \otimes E_{j j}\right) \geq 0$ for all $1 \leq i \leq m, 1 \leq j \leq n$. Applying the same arguments as in the first case on $\psi$, we conclude that $\psi$ satisfies (1) and, hence, $\phi$ satisfies (2). The proof is completed. 


\section{ACKNOWLEDGMENT}

This research was supported by a Hong Kong RGC grant PolyU 502411 with Sze as the PI and Poon as the Co-I. The grant also supported the post-doctoral fellowship of Huang and the visit of Fošner to the Hong Kong Polytechnic University in the summer of 2012. She gratefully acknowledged the support and kind hospitality from the host university. Fošner was supported by

the bilateral research program between Slovenia and US (Grant No. BI-US/12-13-023). Li was supported by a RGC grant and a USA NSF grant; this research was done when he was a visiting professor of the University of Hong Kong in the spring of 2012; furthermore, he is an honorary professor of Taiyuan University of Technology (100 Talent Program scholar), and an honorary professor of the Shanghai University. Poon was supported by a RGC grant and a USA NSF grant.

\section{REFERENCES}

[1] M. Brešar, M. A. Chebotar, W. S. Martindale III, Functional identities, Birkhäuser, Basel (2007).

[2] W.-S. Cheung, C.-K. Li, Y.-T. Poon, Isometries Between Matrix Algebras J. Aust. Math. Soc. 77 (2004), 1-16.

[3] J. Dieudonné, Sur une gén eralisation du groupe orthogonal á quatre variables, Arch. Math. 1 (1949), $282-287$.

[4] A. Fošner, Z. Huang, C.K. Li, and N.S. Sze, Linear preservers and quantum information science, to appear in Linear and Multliinear Algebra, preprint, available at arXiv:1208.1076.

[5] A. Fošner, Z. Huang, C.K. Li, and N.S. Sze, Linear maps preserving Ky Fan norms and Schatten norms of tensor product of matrices, preprint, available at arXiv:1211.0396.

[6] A. Fošner, Z. Huang, C.K. Li, and N.S. Sze, Linear maps preserving numerical radius of tensor product of matrices, preprint, available at arXiv:1212.3134.

[7] G. Frobenius, Über die Darstellung der endlichen Gruppen durch Linear Substitutionen, Sitzungsber Deutsch. Akad. Wiss. Berlin (1897), 994-1015.

[8] H. S. Green, Matrix methods in quantum mechanics, Barnes \& Noble, New York (1968).

[9] K. E. Gustafson and D. K. M. Rao, Numerical range: The Field of values of linear operators and matrices, Universitext, Springer-Verlag, New York (1997).

[10] P. R. Halmos, A Hilbert space problem book, D. Van Nostrand Co., Inc., Princeton (1967).

[11] R.A. Horn and C.R. Johnson, Topics in Matrix Analysis, Cambridge University Press, Cambridge (1991).

[12] N. Johnston, Characterizing Operations Preserving Separability Measures via Linear Preserver Problems, Linear and Multilinear Algebra 59 (2011), 1171-1187.

[13] C.-K. Li, Linear operators preserving the higher numerical radius of matrices, Linear and Multilinear Algebra 21 (1987), 63-73.

[14] C.-K. Li, Matrices with some extremal properties, Linear Algebra Appl. 101 (1988), 255-267.

[15] C.-K. Li, S. Pierce, Linear preserver problems, Amer. Math. Monthly 108 (2001), 591-605.

[16] C.-K. Li, Y.-T. Poon, N.-S. Sze, Linear maps transforming the higher numerical ranges, Proc. Amer. Math. Soc. 133 (2005) 369-377.

[17] C.-K. Li, P. Šemrl, A. R. Sourour, Isometries for Ky-Fan norms on block triangular matrix algebras, Archiv Math. 81 (2003), 175-181.

[18] M. Marcus and I. Filippenko, Nondlfferentiable Boundary Points of the Higher Numerical Range, Linear Algebra Appl. 21 (1978), 217-232.

[19] L. Molnár, Selected preserver problems on algebraic structures of linear operators and on function spaces, Lecture Notes in Mathematics, Vol. 1895, Berlin (2007).

[20] M. Omladič, On operators preserving the numerical range, Linear Algebra Appl. 134 (1990), 31-51.

[21] V. J. Pellegrini, Numerical range preserving operators on a Banach algebra, Studia Math. 54 (1975), $143-147$.

[22] S. Pierce, W. Watkins, Invariants of linear maps on matrix algebras, Linear and Multilinear Algebra 6 (1878), $185-200$.

[23] S. Friedland, C.-K. Li, Y.-T. Poon, N.-S. Sze, The automorphism group of separable states in quantum information theory, J. Math. Phys. 52 (2011), 042203.

[24] Z.-X. Wan, Geometry of matrices, World Scientific Publishing Co., New York (1996). 
Ajda Fošner, Faculty of Management, University of Primorska, Cankarjeva 5, SI-6104 Koper, SLOVEnia

E-mail address: ajda.fosner@fm-kp.si

Zejun Huang, Department of Applied Mathematics, The Hong Kong Polytechnic University, Hung Hom, Hong Kong

E-mail address: huangzejun@yahoo.cn

Chi-Kwong Li, Department of Mathematics, College of William and Mary, Williamsburg, VA 23187, USA; Department of Mathematics, University of Hong Kong, Pokfulam, Hong Kong

E-mail address: ckli@math.wm.edu

Yiu-Tung Poon, Department of Mathematics, Iowa State University, Ames, Iowa 50011, USA

E-mail address: ytpoon@iastate.edu

Nung-Sing Sze, Department of Applied Mathematics, The Hong Kong Polytechnic University, Hung HOM, HONG KONG

E-mail address: raymond.sze@polyu.edu.hk 